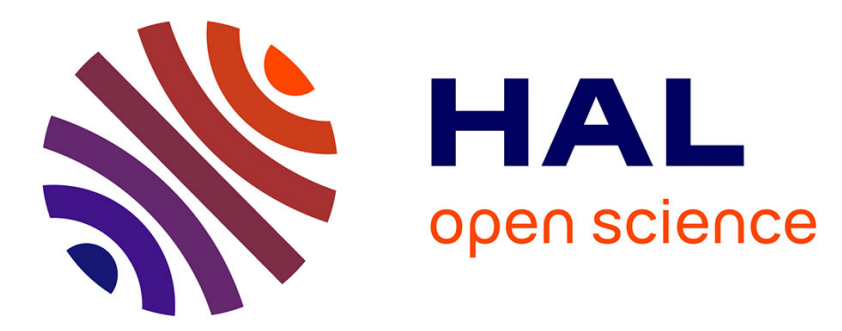

\title{
Multi-objective structural robust optimization under stress criteria based on mixed plate super-elements and genetic algorithms
}

Pierre Garambois, Sébastien Besset, Louis Jézéquel

\section{- To cite this version:}

Pierre Garambois, Sébastien Besset, Louis Jézéquel. Multi-objective structural robust optimization under stress criteria based on mixed plate super-elements and genetic algorithms. Structural and Multidisciplinary Optimization, 2015, 10.1007/s00158-015-1339-4 . hal-01266872

\section{HAL Id: hal-01266872 \\ https://hal.science/hal-01266872}

Submitted on 7 Feb 2016

HAL is a multi-disciplinary open access archive for the deposit and dissemination of scientific research documents, whether they are published or not. The documents may come from teaching and research institutions in France or abroad, or from public or private research centers.
L'archive ouverte pluridisciplinaire HAL, est destinée au dépôt et à la diffusion de documents scientifiques de niveau recherche, publiés ou non, émanant des établissements d'enseignement et de recherche français ou étrangers, des laboratoires publics ou privés. 
Noname manuscript No.

(will be inserted by the editor)

\section{Multi-objective structural robust optimization under stress criteria based on mixed plate super-elements and genetic algorithms}

\author{
Pierre Garambois . \\ Sébastien Besset . \\ Louis Jézéquel
}

Received: date / Accepted: date simplicity, saves computational time and the Paretofront presentation with stress objective provides a good overview of the possibilities for the designers.

Keywords Multi-objective optimizations · Plate Structural Dynamic · Mixed Finite Element Model (MFEM) · Mixed Super-Element (MSE) · substructuring modal methods $\cdot$ stress criteria

\section{Introduction}

The goal of this paper is to present a new methodology for plate structural dynamic Multi-Objective (MO) optimization with thickness parameters along the structure and stress criteria, using Genetic Algorithms (GA). The idea is to implement a Mixed Finite Element Model (MFEM) based on a displacement-stress mechanical formulation, enhanced with a sub-structuring reduction method. It results a Mixed Super-Element (MSE) featured by a direct access to the stress and a small numerical size, which improves the work of the GA.

Even though often carried out manually, structural dynamic MO optimization methods are developed in the literature, such as gradient [1,25] and MO Evolutionary Algorithms(EA) methods [24,27]. MOEA permit to dispense with any criteria weighting. In this field, many methods are available 20] such as Strength Pareto Evolutionary Algorithms (SPEA [41,39] and SPEAII [40]), Pareto Archived Evolution Strategy (PAES [23]), Non-dominated Sorting Genetic Algorithms (NSGA [33] and NSGA-II [10,11]) or Adaptative Pareto Algorithm (APA 14,13]). In this study, the purpose is not, strictly speaking, to work on the algorithms, but to develop a tool usable by any MOEA. We chose to perform the optimization with a NSGA-II method, because it appeared to be a simple and common method. These methods lead to a set of Pareto-optimal solutions, but the inconvenience lies in the repetitions of the criteria's evaluation. The main idea of this work is to find a way to reduce the CPU-Time necessary for this evaluation.

Some of the previous works on thin structures optimization focus on laminated composites [1] 27], shell 25,8 and piezoelectric smart structures 24. In these cases, the calculation of stresses is mostly done as a constraint, and rarely as an objective like we do. Furthermore, they use primal displacement FEMs, that require extra calculations to get the stress, and rebuilding of the meshing to change the parameters. Our work is based on the use of a different FEM mechanical formulation. Among those available in the literature [37, the Hellinger-Reissner's (HR) 21, 29, 30, 31, that describes displacements and stresses, has been chosen for our 
problems. The use of mixed theorems has been popularized in the context of plate structures analysis, because it provides computational advantages for such representation. Many MFEMs have been implemented over the years for different theories such as $3 \mathrm{D}$ representations [28, 2D [19,34,35, plates 34,35, 12,9,32, and multilayered and piezoelectric structures [5, 6, 2, 17, 18. Wriggers' book 38. provides a wide range of application for MFEMs. Besides the computational interests for various mechanical elastic theories, MFEMs give the big advantage of providing stress parameters at the master of the element level with the response of the structure. In this way, we program a Dynamic Mixed FEM (DMFEM), for Kirchoff-Love (KL) thin plates. This model allows a faster access to the stress fields, one objective of the optimization. Furthermore, the implementation of the plate theory permits to act on the thicknesses without building a new assembly which improves the GA's performances since thicknesses of each zones are parameters of the optimization.

The DM-FEM presents a detrimental feature because of its bigger size, which increases the CPU-Time of the structure response under a dynamic load. In this way, we decide to implement a sub-structuring reduction method in order to reduce the DM-FEM splitting the structure into reduced ones with their own thicknesses. Sub-structuring methods exist so as to reduce primal FEM, such as "fixed interface mode" methods [7, "free interface mode" methods [26] and "boundary mode" methods [36, 4. The principle of those methods is to split the structure into a few sub-structures, and to express the behavior of each with its own chosen eigenmodes. These methods have also been associated to build up a Double Modal Synthesis [22,3. with both sub-structures and junctions condensation. An adaptation of these displacement methods for MFEM has been imagined by the authors [15,16]. The principle is to build, for each sub-structure in parallel, a reduced basis, for the displacements with the existing primal methods, and a projection of the primal basis for the stresses. New mixed reduced basis for each sub-structure are then built up with modes from the primal model. The sub-structures are linked through physical Degrees Of Freedoms (DOFs) kept in the new basis. That method reduces the number of DOF [15] and the CPU-Time for the global structure response, and keeps the advantages of the MFEM. In this MO-optimization, each substructure has a constant thickness and thus one single parameter. That reduction is linearly dependent from this parameter and turns each zone into a MSEs, whose thickness is mutable each iteration without rebuilding. The evaluation of the response is then computed each iteration on the reduced assembled DM-FEM.
In the first part, we present the optimization problem and the GA. We continue with the implementation of the DM-FEM and the sub-structuring method, highlighting the differences with regular methods. Finally we present a few investigations, made with the GA-based MO optimization powered by the MSEs. The test structure is splitted into sub-structures (MSEs) with one thickness each (parameters), and we minimize the mass and the Von Mises (VM) stress within the whole plate (objectives) under dynamic loads (both discrete and continuous frequency band are presented). The method is able to satisfy multiple stress criteria with different thickness distributions, it saves CPU-Time, and the Pareto-front presentation with a stress objective gives a good overview of the design possibilities.

\section{Optimization problem and use of Genetic Algorithms}

The "example" structure we want to optimize is built up with 768 thin plate elements (see figure 1). It is composed of 3 flat sub-structures, with their own thicknesses, parameters of the optmimization (range defined by the user).

The problem we try to solve is the following: we want to minimize both the maximum VM stress within the whole plate structure under a harmonic load and the mass in a bid of costs. We consider two different cases (see figure 1): a frequency equal to an eigenfrequency of the system, and a frequency that belong to a frequency band in a matter of robustness. The two objectives being antagonistic, the goal is to find some thickness distributions that lead good compromises between the mass and the maximum stress undergone by the structure. As such, MO-GA-based methods quite naturally appears.

The GA methods are based on the evolution of species in their natural environment. The functioning of the algorithm is summarized in figure 2). We consider "individuals" that are solutions to the problem (here, a combination of 3 thicknesses) and that give compromises between the objectives. A group of individuals constitutes a "population" (here, a set of thickness combinations, that forms a Pareto front with one compromise per thickness distribution). The goal of the GA consists in making evolve the population over the "generations" (iterations) by doing mutations and crossing between the individuals of this population, in order to improve for the purpose of our objectives regarding these individuals. The GA is composed of 5 different stages (see figure 2). The italic descriptions in figure 2 represent the adaptation of the GA to our problem. 
The main contributions and innovations of our work appear in the grey blocks (figure 2). We improve the Evaluation step in terms of CPU-time and complexity, and add a preliminary step 0 .

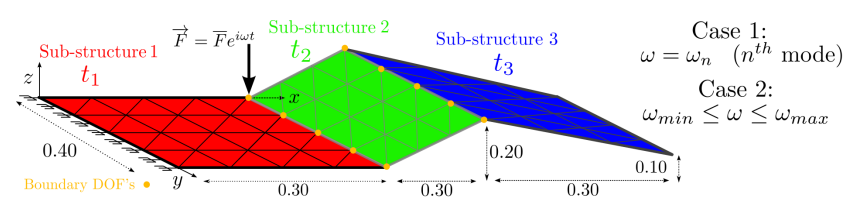

Fig. 1: Plate structure made of 3 flat sub-structures with their own thicknesses

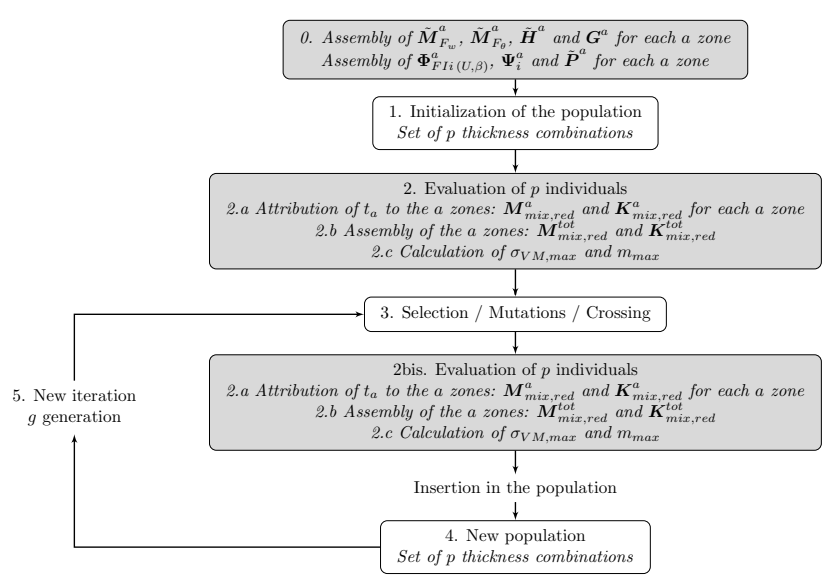

Fig. 2: Principle of the genetic algorithm with $p$ individuals and $g$ generations

\section{Mixed Finite Element Model for thin Kirchhoff-Love plate}

The "evaluation step" of the GA (step 2 in figure 2) is the most costly because we need to evaluate the stress within the whole structure, and identify the maximum. The classical method consists in building a primal FEM to get the displacements and use extra calculations to reach the stress. It is the most common and intuitive method, but it appears to be heavy going because of the extra calculations. Our method build a displacementgeneralized stress DM-FEM for plates that gives a direct access to both fields. Another important benefit, when choosing a plate theory, is the possibility of modifying the thickness as a parameters without rebuilding a whole assembly.

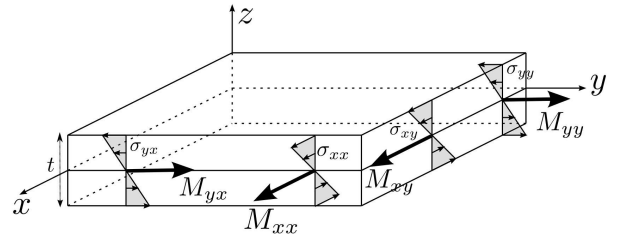

(a)

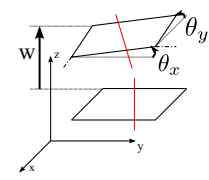

(b)
Fig. 3: Kirchhoff-Love theory: (a) Generalized stresses (b) Displacements

This type of DM-FEM is based on the HR mixed functional [31,38] expressed for dynamics mechanical problems as follows:

$$
\begin{array}{r}
\Pi_{H R D}=\iiint_{V}-\boldsymbol{\sigma}_{i j} \boldsymbol{e}_{i j}\left(\boldsymbol{u}_{i}\right)+\frac{1}{2} \boldsymbol{\sigma}_{i j} \boldsymbol{S}_{i j k l} \boldsymbol{\sigma}_{k l}+ \\
\boldsymbol{b}_{i} \boldsymbol{u}_{i}+\frac{1}{2} \rho \dot{\boldsymbol{u}}_{i}^{2} \mathrm{dV}
\end{array}
$$

considering $\boldsymbol{\sigma}_{i j}$ the stress, $\boldsymbol{u}_{i}$ the displacement, $\boldsymbol{e}_{i j}\left(\boldsymbol{u}_{i}\right)$ the strain function of the displacement $\boldsymbol{u}_{i}, \boldsymbol{b}_{i}$ the body force, $\rho$ the density and $\boldsymbol{S}_{i j k l}$ the elastic compliance matrix. The Euler-Lagrange equations can then be applied to the functional so as to conventionally solve a dynamic structure problem.

The discretization of the generalized stresses and the displacements, with plate elements (thickness $t$ ) using Kirchhoff-Love (KL) theory (see figure 3) is as follows:

$\boldsymbol{\sigma}_{i j}=\left\{M_{x}, M_{y}, M_{x y}\right\}^{T}=\boldsymbol{P} \boldsymbol{\beta}$

$\boldsymbol{u}_{i}=\left\{w, \theta_{x}, \theta_{y}\right\}^{T}=\mathbf{N} \boldsymbol{U}$

$\boldsymbol{e}_{i j}=\left\{\epsilon_{x x}, \epsilon_{y y}, \gamma_{x y}\right\}^{T}=\boldsymbol{D} \boldsymbol{u}_{i}=\boldsymbol{D N} \boldsymbol{U}$

with $\left\{M_{x}, M_{y}, M_{x y}\right\}^{T}$ respectively the bending moments in the $x$ and $y$ direction, and the twisting moment within the plate, $\left\{w, \theta_{x}, \theta_{y}\right\}^{T}$ respectively the transverse displacement of the plate, and the section rotations around $x$ and $y . \boldsymbol{P}$ is the generalized stresses shape function matrix with $\boldsymbol{\beta}$ the generalized stress parameters vector, $\boldsymbol{N}=\left\{\boldsymbol{N}_{w}, \boldsymbol{N}_{\theta}\right\}^{T}$ the displacements shape function matrix, $\boldsymbol{U}=\left\{\boldsymbol{U}_{w}, \boldsymbol{U}_{\theta}\right\}^{T}$ the displacements parameters vector and $\boldsymbol{D}$ the displacement-strain operator.

When carefully splitting the matrices dependent on $t, t^{3}$ and $\frac{1}{t^{3}}$, the Euler-Lagrange equations leads to the following matrix formulation of the DM-FEM (for one 
plate element of surface $S)$ :

$$
\begin{aligned}
& \underbrace{\left\{\begin{array}{ccc}
t \tilde{\boldsymbol{M}}_{F_{w}} & \mathbf{0} & \mathbf{0} \\
\mathbf{0} & t^{3} \tilde{\boldsymbol{M}}_{F_{\theta}} & \mathbf{0} \\
\mathbf{0} & \mathbf{0} & \mathbf{0}
\end{array}\right\}}_{\boldsymbol{M}_{m i x}}\left\{\begin{array}{c}
\ddot{\boldsymbol{U}}_{F_{w}} \\
\ddot{\boldsymbol{U}}_{F_{\theta}} \\
\ddot{\boldsymbol{\beta}}
\end{array}\right\}+ \\
& \underbrace{\left\{\begin{array}{ccc}
\mathbf{0} & \mathbf{0} & \boldsymbol{G}_{w}^{T} \\
\mathbf{0} & \mathbf{0} & \boldsymbol{G}_{\theta}^{T} \\
\boldsymbol{G}_{w} & \boldsymbol{G}_{\theta} & \frac{1}{t^{3}} \tilde{\boldsymbol{H}}
\end{array}\right\}}_{\boldsymbol{K}_{m i x}}\left\{\begin{array}{c}
\tilde{U}_{F_{w}} \\
\boldsymbol{U}_{F_{\theta}} \\
\boldsymbol{\beta}
\end{array}\right\}=\left\{\begin{array}{c}
\boldsymbol{F}_{w} \\
\boldsymbol{F}_{\theta} \\
\mathbf{0}
\end{array}\right\}
\end{aligned}
$$

with $\quad \tilde{\boldsymbol{S}}_{i j k l}=t^{3} \boldsymbol{S}_{i j k l}, \quad \tilde{\boldsymbol{M}}_{F_{w}}=\iint_{S} \boldsymbol{N}_{\boldsymbol{w}}^{T} \rho \boldsymbol{N}_{\boldsymbol{w}} \mathrm{dS}$, $\tilde{\boldsymbol{M}}_{F_{\boldsymbol{\theta}}}=\iint_{S} \boldsymbol{N}_{\boldsymbol{\theta}}^{T} \frac{\rho}{12} \boldsymbol{N}_{\boldsymbol{\theta}} \mathrm{dS}, \quad \boldsymbol{G}_{\boldsymbol{w}}=\iint_{S} \boldsymbol{P}^{T} \boldsymbol{D} \boldsymbol{N}_{\boldsymbol{w}} \mathrm{dS}$, $\boldsymbol{G}_{\boldsymbol{\theta}}=\iint_{S} \boldsymbol{P}^{T} \boldsymbol{D} \boldsymbol{N}_{\boldsymbol{\theta}} \mathrm{dS}, \quad \tilde{\boldsymbol{H}}=\iint_{S}-\boldsymbol{P}^{T} \tilde{\boldsymbol{S}}_{i j k l} \boldsymbol{P} \mathrm{dS}$, $\boldsymbol{G}=\left\{\boldsymbol{G}_{w}, \boldsymbol{G}_{\theta}\right\}$ and $\boldsymbol{F}=\left\{\boldsymbol{F}_{w}, \boldsymbol{F}_{\theta}\right\}^{T}$ the nodal forces (splitted $w / \theta)$.

The first benefit of this model compared to displacement ones is the direct access to the stress parameters. In fact, it doesn't require to rebuild the stress field from the displacements like we do with a displacement FEM, and improve the stress evaluation.

The second advantage is the thickness independence of the matrices (equations 5. Basically, it means that the model doesn't require to rebuild $\tilde{\boldsymbol{M}}_{F_{w}}, \tilde{\boldsymbol{M}}_{F_{\theta}}, \boldsymbol{G}$ and $\tilde{\boldsymbol{H}})$ for the same meshing, and allows to modify the thickness parameters each iteration of the optimization with an almost instantaneous operation.

Nevertheless, it has the inconvenience of being bigger than a displacement FEM (see Table 1), which, on the other hand, may slow down the computation of the structure response. A sub-structuring reduction method is thus implemented, in order to decrease the size of the DM-FEM, and keeping the thickness independence of the method and the stress access.

\section{Sub-structuring method}

The reduction method used in this paper has been explained in [15, 16]. The idea is to split the structure into few sub-structures, and reduce each of them separately in parallel, using the primal corresponding FEM, turning them into MSE. Then we assemble them and compute the stress on the global reduced structure.

The initial Degrees Of Freedom (DOF) of a given substructures $a$ are separated in internal DOF $\boldsymbol{U}_{i}^{a}$, junction DOF $\boldsymbol{U}_{j}^{a}$ (allowing to link sub-structures between them) and stress DOF $\boldsymbol{\beta}^{a}$. The displacements are projected on a basis composed of truncated primal "fixed" modes (see Craig \& Bampton method [4]) and the stresses on a basis composed of truncated primal "fixed" modes as well, but projected on the stresses. The new reduced parameters are then: truncated modal components $\boldsymbol{\eta}_{F I(U, \beta)}^{a}$, and the junction DOF $\boldsymbol{U}_{j}^{a}$ remaining unchanged. The reduction of the whole sub-structure $a$ is given by:

$$
\begin{aligned}
& \left\{\begin{array}{c}
\boldsymbol{U}_{i}^{a} \\
\boldsymbol{U}_{j}^{a} \\
\boldsymbol{\beta}^{a}
\end{array}\right\}= \\
& \left\{\begin{array}{c}
\boldsymbol{\Phi}_{F I i(U, \beta)}^{a} \\
\mathbf{0} \\
t^{3} \tilde{\boldsymbol{P}}^{a}\left\{\begin{array}{c}
\boldsymbol{\Phi}_{F I i(U, \beta)}^{a} \\
\mathbf{0}
\end{array}\right\} t^{3} \tilde{\boldsymbol{P}}^{a}\left\{\begin{array}{c}
\boldsymbol{\Psi}_{i}^{a} \\
\boldsymbol{I}_{i j}
\end{array}\right\}
\end{array}\right\}\left\{\begin{array}{c}
\boldsymbol{\eta}_{F I(U, \beta)}^{a} \\
\boldsymbol{U}_{j}^{a}
\end{array}\right\}
\end{aligned}
$$

with $\tilde{\boldsymbol{P}}^{a}=-\left(\tilde{\boldsymbol{H}}^{a}\right)^{-1} \boldsymbol{G}^{a}, \boldsymbol{\Phi}_{F I i(U, \beta)}^{a}$ being a truncated basis of the "fixed" modes of the sub-structures $a$ (junction considered fixed) and $\boldsymbol{\Psi}_{i}^{a}=-\boldsymbol{K}_{\boldsymbol{i} \boldsymbol{i}}{ }^{-1} \boldsymbol{K}_{\boldsymbol{i} \boldsymbol{j}}$ being the matrix of the constraint static modes, taken from the primal FEM. We assemble two sub-structures $a$ and $b$ considering: $\boldsymbol{U}_{j}^{a}=\boldsymbol{U}_{j}^{b}=\boldsymbol{U}_{j}$.

When reducing flat sub-structures, the formulation of the reduced basis gives other advantages: the "fixed" modes of the sub-structure $a \boldsymbol{\Phi}_{F I i(U, \beta)}^{a}$ remains the same, even when the thickness change and the basis $\left\{\boldsymbol{\Psi}_{i}^{a}, \boldsymbol{I}_{i j}\right\}^{T}$ (representing the reaction of the sub-structure when its junction is moving) doesn't depend on the thickness of the plate. In this way, our reduction method is all the more robust that it is easily transposable to a different thickness of one sub-structure (doesn't requires to rebuild the matrices $\boldsymbol{\Phi}_{F I i(U, \beta)}^{a}, \boldsymbol{\Psi}_{i}^{a}$ and $\tilde{\boldsymbol{P}}^{a}$ of each sub-structure $a$ built in step 0 ).

Basically, each sub-structure works as a MSE, defined by its thickness only. Each individual of each generation, a simple operation is made in parallel for each MSE $a$, in order to modify their thicknesses (colored in red) in the matrices of equation 5 and 6 (step 2.a figure 2). Then we assemble the global matrices $\boldsymbol{M}_{\text {mix, red }}^{\text {tot }}$ and $\boldsymbol{K}_{\text {mix,red }}^{\text {tot }}$ (step 2.b figure 2). Then, the step 2.c figure2 is done with the computation of the structure response on the reduced global model as follows:

$$
\left\{\begin{array}{l}
\overline{\boldsymbol{U}} \\
\overline{\boldsymbol{\beta}}
\end{array}\right\}=\left(-\omega^{2} \boldsymbol{M}_{\text {mix }, \mathrm{red}}^{\mathrm{tot}}+(1+i \xi) \boldsymbol{K}_{\text {mix }, \mathrm{red}}^{\text {tot }}\right)^{-1}\left\{\begin{array}{c}
\overline{\boldsymbol{F}} \\
0
\end{array}\right\}
$$

with $\xi$ the hysteretic damping coefficient.

Then, the maximum VM stress $\sigma_{V, M, \max }$ is easily accessible thanks to the mixed feature:

$\sigma_{V M}=\frac{1}{\sqrt{2}} \sqrt{\left(\sigma_{x x}-\sigma_{y y}\right)^{2}+\sigma_{y y}^{2}+\sigma_{x x}^{2}+6 \sigma_{x y}^{2}}$

Table 1) summarizes the benefits of our MSEs in terms of time gain, comparing the Dynamic Primal FEM, the 
Dynamic Mixed FEM and the Reduced Dynamic Mixed FEM.

Table 1: Methods Characteristics - 768 elements, 3 MSEs, 20 modes each

\begin{tabular}{cccc}
\hline FEM & Primal & Mixed & Reduced Mixed \\
\hline Field & $U$ & $U, \beta$ & $U, \beta$ \\
Stress field & Rebuild & Direct & Direct \\
DOFs & 2448 & 9360 & 168 \\
Time Step 2 $(\mathrm{s})$ & 3.00 & 2.50 & 2.15 \\
\hline Gain & Ref & $16.7 \%$ & $28.2 \%$ \\
\hline
\end{tabular}

\section{Example: optimization of the thickness distribution to minimize the mass \& the maximum stress}

The example we use is an academic simple structure schematized in figure 1 in section 2). The FEM representation makes the method transposable to an industrial case. We minimize the maximum VM stress within the structure and the mass. The parameters are the thicknesses of each MSE or "sub-structure" (thickness zones in figures 1 and 4 c and 5 c). The thickness parameters may vary in a range between 1 and $2 \mathrm{~mm}$. We consider $\rho=7500 \mathrm{~kg} / \mathrm{m}^{3}, E=210 G P a$ and $\nu=0.33$. The pulsation studied will be both discrete (subsection 5.1) and within a frequency band (5.2). Table 1 shows the improvements of this method compared to classical ones in terms of time gain.

The results are presented as "Pareto Front" (black points in 4a, 5a and 6a). These points are defined by the right ordinate axis and the abscissa axis representing respectively the mass of the structure and the maximum VM stress. Each of these optimal Pareto-points are better than the others in the sense of at least one of the two objectives and are compromises we can select to design our structure, depending on the objective we want privilege. Once a Pareto point chosen, the colored curves in 4a, 5a and 6a lead to the parameters corresponding to the point. They are defined by the left ordinate axis and the abscissa axis representing respectively the thickness of each of the sub-structures for the chosen Pareto point (with the same abscissa) and the maximum VM stress corresponding to this point. Basically, when selecting a point of the Pareto Front, the corresponding thicknesses to design the structure correspond to ordinate of the colored three points with the same abscissa. This kind of presentation with stress criteria and thickness curve provides a good overview of the possibilities for the user.

Those results were obtained with NSGA-II (population of 100 individuals and 1000 generations) and the method described in the last sections. The GA has been compiled several times so as to check the convergence of the results.

The first subsection deals with the discrete frequency case for the mode 1 (intuitive result) and 7 (more complex), whereas the second one treats of a more robust case with a frequency band $(70-100 \mathrm{~Hz})$.

\subsection{Discrete Frequency: $\omega=\omega_{n}, n^{\text {th }}$ mode \\ 5.1.1 Simple example: mode $1, \xi=1 e-5$}

The results presented in figure $4 a, b, c$ show some thickness combinations allowing a VM stress between 21 and $89 \mathrm{MPa}$, with a mass of the structure between 2.9 and 4.7 Kgs. In this case, more than the Pareto-Front itself, the most relevant is the evolution of the thicknesses in function of VM stress (colored curves in figure 4a). We observe that the most important zone to "reinforce" is the first one, and then the second one. When taking a look at the stress distribution for this mode (figure $4 \mathrm{~b}$ ), that evolution of thicknesses in function of the stress seems coherent as we would intuitively decrease the thickness where the stress is low, and increase it where it is high. This test puts forward the qualitative validity of the method, and brings a quantitative point stress-wise.

\subsubsection{Test example: mode $7, \xi=1 e-3$}

The results are presented in figure 5a,b,c. Considering the stress distribution for the mode 7 (figure 5b), the results are not intuitive. This example shows some thickness combinations allowing a VM stress between 18 and $140 \mathrm{MPa}$, with a mass of between 2.9 and $4.7 \mathrm{Kgs}$. The evolution of the 3 parameters in function of VM stress (colored curves in figure $5 \mathrm{a}$ ) is very specific. In fact, we distinguish 2 main zones. From 18 until $86 \mathrm{MPa}$ : it is the lowest stress range. It appears that the third zone is stuck to his minimum whereas the two others has influence. The latters have a decreasing thickness as the VM stress increases, and the first zone is more important than the second one.

From 86 until 140 MPa: it is the highest stress range. The evolution of the thicknesses and the order of magnitude are different. The three parameters have close values (between 1 and $1.3 \mathrm{~mm}$ ) and their evolution is 


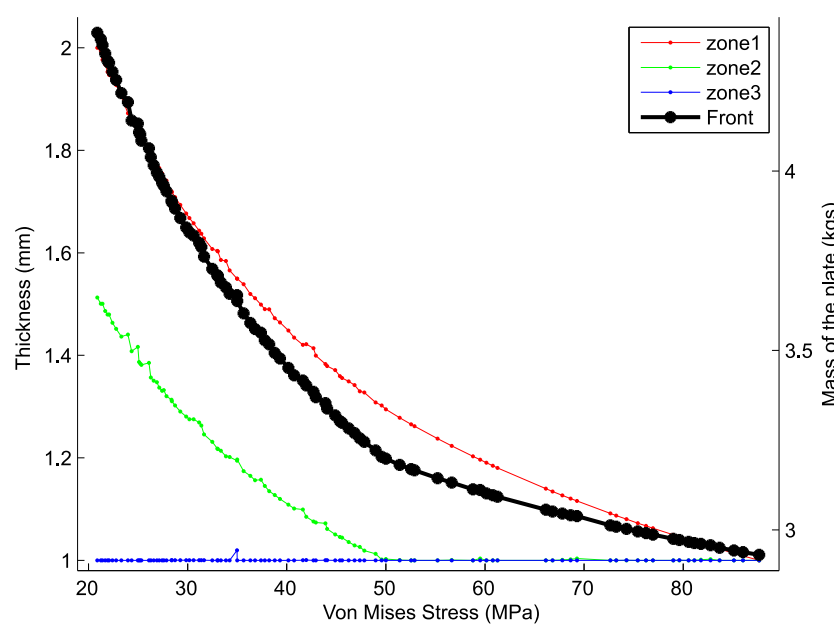

(a)

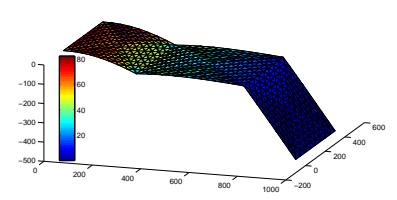

(b)

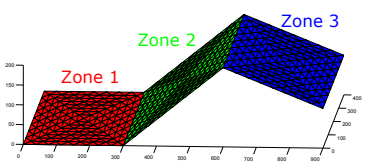

(c)
Fig. 4: Results case 1 for mode 1: (a) Pareto Front (black) \& thicknesses (color), (b) Stress distribution (MPa) on the mode, (c) Thickness zones

close as well, decreasing the same from 1.3 till $1 \mathrm{~mm}$ as the VM stress increases.

Those results define a phenomenon we call "parametric typing". Although the Pareto Front has no singularities, the parameters' evolution do not follow any specific or logical rule. Nevertheless, they can be divided into two main stress domains which correspond to different thickness distributions. Interesting advantage in terms of design possibilities: you can find two different thickness distributions (two designs) that leads to close optimums (close properties regarding the objectives) at the junction of the two zones. Those results could be interesting with more complex industrial cases, and puts forward another interest of the optimization, in addition to the efficiency previously highlighted.

\subsection{Frequency band: $\omega_{\min } \leq \omega \leq \omega_{\max },, \xi=1 e-3$}

For the sake of robustness, this section focuses on a harmonic excitation whose pulsation varies in a frequency band $70-100 \mathrm{~Hz}$. For each thickness distribution, the VM stress has to be calculated for all the modes whose pulsation is within the frequency band (equations 7 and 8 for each mode) in order to find the more constraint modes. Nevertheless, since the eigenfrequencies varies

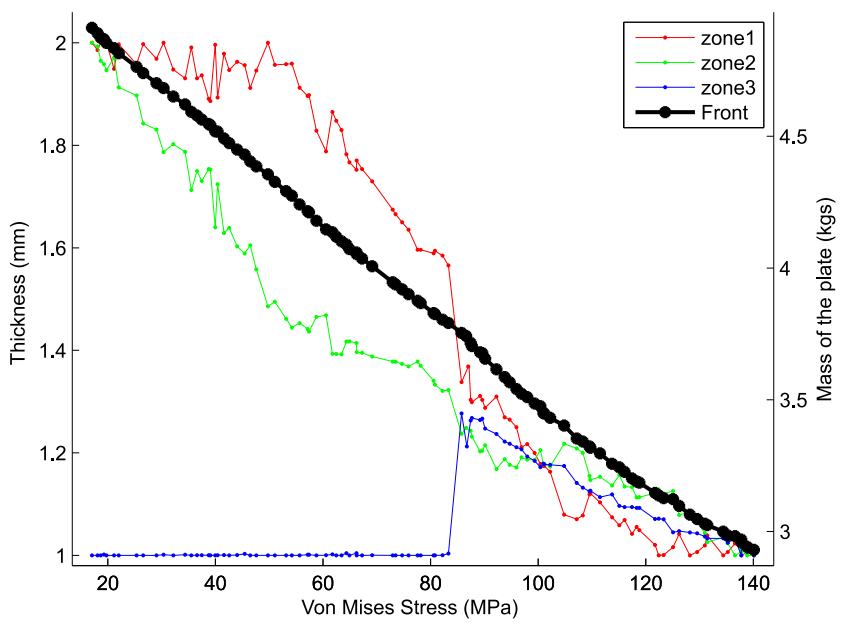

(a)

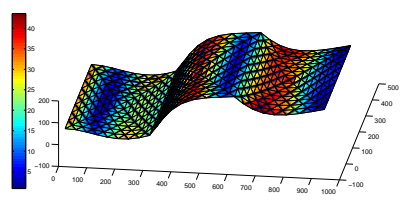

(b)

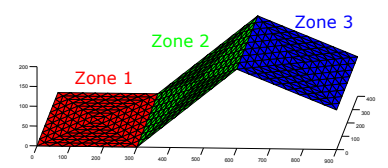

(c)
Fig. 5: Results case 1 for mode 7: (a) Pareto Front (black) \& thicknesses (color), (b) Stress distribution (MPa) on the mode, (c) Thickness zones

with the parameters, one can disappear from the frequency band, and let the maximum VM stress to another mode. The results presented in figure 6 show that 4 modes influence the optimization (11 to 14 , shape form in figure 6 b,c,d,e), and successively present the highest VM stress. The horizontal arrows define the concerning mode in each domain, and the frequency band associated. The domains 1 and 4 present the particularity of mixing the influence of two modes (respectively $11 / 12$ and 13/14) whereas the domains 2,3 and 5 deals with the same mode (respectively 12, 13 and 14). This alternation result in a singular Pareto Front (figure 6a).

The domain 1 (low stress) is mass-typed as all the points have close masses, and the 3 MSEs' thicknesses have the same order of magnitude. As the stress increases, the influence of the third zone decreases, the second one increases and the first one looks stable. We also observe a mass "gap" with the next domain.

The domains 2 and 3 (middle stress) present an interesting singularity as the thickness of the $3^{r d}$ MSE remains in the highest range $(1.8-2.0 \mathrm{~mm})$ whereas the two others remain minimum.

The domains 4 and 5 present a classical Pareto-front with different thickness parameters distributions and 
order of magnitude. The MSEs' thickness 3 remains the highest whereas the zones 1 and 2 are no longer minimum in the domain 4 , and minimum again in domain 5.

As explained in the last case, the singularities in the thickness values (parametric typing) and the Pareto front present designs benefits. For example the junction between the zone 3 and 4 presents two close point objectives-wise with two different shapes. The junction between domains 1 and 2 present two close points stress-wise with very different shapes as well. That domain 1 also highlights a common problem in structural mechanics when making the structure, because few points with close parameters (shapes) show high differences in terms of objectives (stress in this case).

\section{Conclusion}

This paper introduces a new methodology for MO optimization of structural dynamic plate problems with stress criteria and thickness parameters. The optimization is made with a classical NSGA-II method that finds a set of Pareto-optimal solutions, and presents the originality of using MSEs built up with a displacement-stress DM-FEM for plates enhanced with an adapted substructuring reduction method. The mixed features provides a direct access to the stress objective, the plate theory prevents from rebuilding each iteration while changing the thickness parameters, and the sub-structuring method reduces the CPU-time of the structure response. In the case of 3 MSEs, 768 finite elements and a reduction with 20 modes each MSE, the method is $28.2 \%$ faster than a classical method using plate displacement FEM. The MO optimization's goal is to minimize the maximum VM stress and the mass of the plate structure in function of the thickness of each sub-structure, under a dynamic load with both discrete frequencies and frequency bands. Besides the computational interest, the results have shown interesting singularities in both the evolution of the Pareto-Front and the parameters in function of the stress objective. Parametric typings put forward good designs possibilities and the MO Pareto-Front presentation provides a quick overview of the different options for the designer. In the end, it would be relevant to investigate deeply on the MOEA in order to improve the algorithm part of the optimization and save even more CPU-Time.

\section{References}

1. A.L. Araujo, P. Martins, C.M. Mota Soares, Mota Soares C.A., and J. Herskovits. Damping optimization of

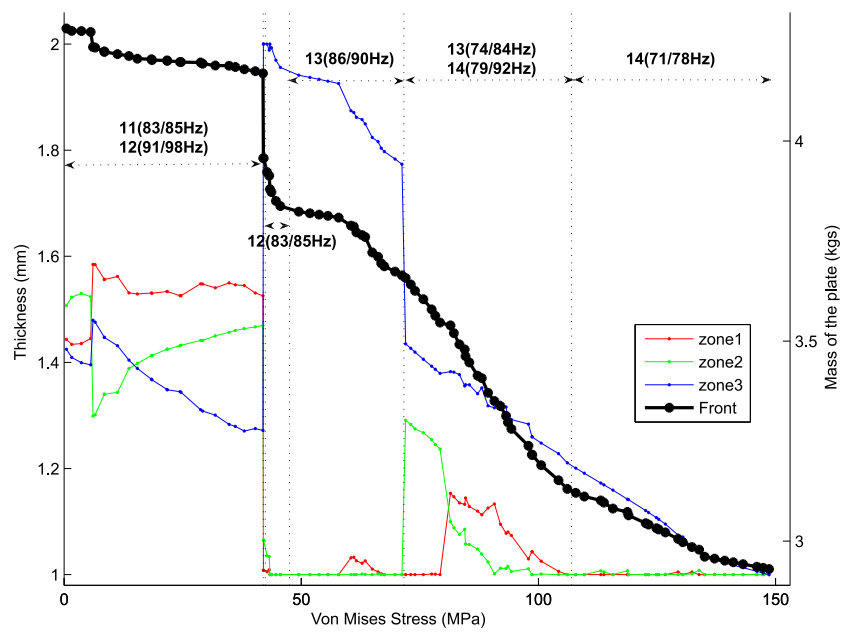

(a)

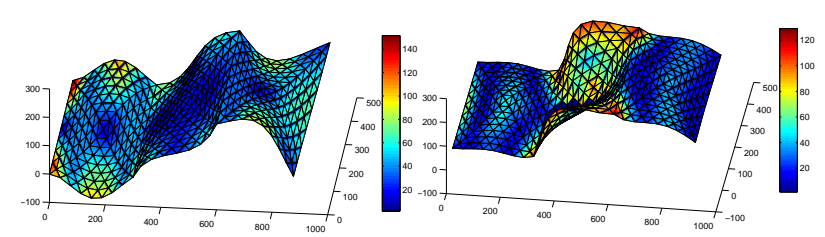

(b)

(c)

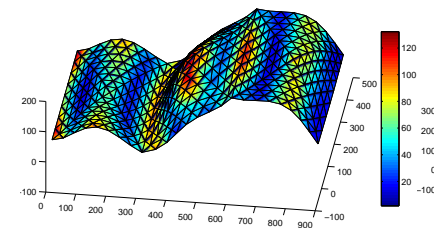

(d)

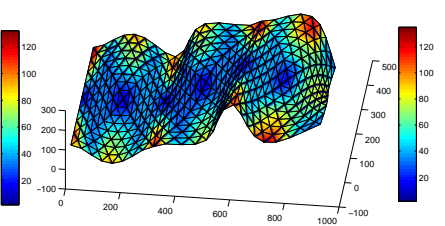

(e)
Fig. 6: Results case 2 for the frequency band $70-100 \mathrm{~Hz}$ : (a) Pareto Front (black) \& thicknesses (color), (b-e) Stress distribution (MPa) on modes 11-14

viscoelastic laminated sandwich composite structures. Structural and Multidisciplinary Optimization, 39:569579, 2009.

2. A. Benjeddou. Advances in piezoelectric finite element modeling of adaptative structural elements: a survey. Computers and Structures, 76:347-363, 2000.

3. S. Besset and L. Jézéquel. Dynamic sub-structuring based on a double modal analysis. Journal of Vibration and acoustics, 130(1):011008, 2008.

4. D. Brizard, L. Jézéquel, S. Besset, and B. Troclet. Determinental method for locally modified structures. application to the vibration damping of a space launcher. Computational Mechanics, 20:631-644, 2012.

5. E. Carrera and L. Demasi. Classical and advanced multilayered plate elements based upon pvd and rmvt. part 1: Derivation of finite element matrices. International Jour- 
nal for Numerical Methods in Engineering, 55:191-231, 2002.

6. E. Carrera and L. Demasi. Classical and advanced multilayered plate elements based upon pvd and rmvt. part 2: Numerical implementation. International Journal for Numerical Methods in Engineering, 55:253-291, 2002.

7. R.R. Craig and M.C.C. Bampton. Coupling of substructures for dynamic analysis. AIAA Journal, 6(7):13131319, 1968.

8. L. Dai, Z.Q. Guan, B.S. Chen, and H.W. Zhang. An open platform of shape design optimization for shell structures. Structural and Multidisciplinary Optimization, 35:609$622,2008$.

9. S. de Miranda and F. Ubertini. A simple hybrid stress element for shear deformable plates. International Journal for Numerical Methods in Engineering, 65:808-833, 2006.

10. K. Deb, S. Agrawal, A. Pratap, and T. Meyarivan. A fast elitist non-dominated sorting genetic algorithm for multi-objective optimization: Nsga-2. Notes in Computer Science, 2000.

11. K. Deb, A. Pratap, S. Agrawal, and T. Meyarivan. A fast and elitist multiobjective genetic algorithm: Nsga- 2 . Transactions on evolutionary computation, 6 (2), 2002.

12. M. Duan, Y. Miyamoto, S. Iwasaki, and H. Deto. Numerical implementation of hybrid-mixed finite element model for reissner-mindlin plates. Finite Element in Analysis and Design, 33:167-185, 1999.

13. D. Dumitrescu, C. Groşan, and M. Oltean. A new evolutionary adaptative representation paradigm. Studia Universitas Babes-Boylai, Seria Informatica, XLVI(1):15-30, 2001.

14. D. Dumitrescu, C. Groşan, and M. Oltean. Simple multiobjective evolutionary algorithm. In Seminars on Computer Science, Faculty of Mathematics and Computer Science, Babes-Boylai University of Cluj-Napoca, 2001.

15. P. Garambois, S. Besset, and L. Jézéquel. Modal synthesis applied to a reissner mixed plate finite element dynamic model. Proceedings of the 9th International Conference on Structural Dynamics, EURODYN 2014, 2014.

16. P. Garambois, S. Besset, and L. Jézéquel. Various double component mode synthesis and sub-structuring methods for dynamic mixed fem. European Journal of Mechanics - A/Solids, 53:196-219, 2015.

17. R. Garcia Lage, C.M. Mota Soares, C.A. Mota Soares, and J.N. Reddy. Analysis of adaptative plate structures by mixed layerwise finite elements. Composite Structures, 66:269-276, 2004.

18. R. Garcia Lage, C.M. Mota Soares, C.A. Mota Soares, and J.N. Reddy. Modelling of piezolamited plates using layerwise mixed elements. Computers and Structures, 82:1849-1863, 2004.

19. M. Gellert and M.E. Laursen. Formulation and convergence of a mixed finite element method applied to elastic arches of arbitrary geometry and loading. Computer Methods In Applied Mechanics and Engineering, 7:285302, 1976 .

20. C. Groşan and D. Dumitrescu. A comparison of multiobjective evolutionary algorithms. Acta Universitatis Apulensis, 4, 2002.

21. E. Hellinger. Die allgemeinen ansatze der mechanik der kontinua. Encyklopadie der mathematischen Wissenschaften, 4, 1914.

22. L. Jézéquel and H.D. Setio. Component modal synthesis methods based on hybrid models, part 1: Theory of hybrid models and modal truncation methods. Journal of Applied mechanics, 61:100-108, 1994.
23. J.D. Knowles and D.W. Corne. The pareto archived evolution strategy: A new baseline algorithm for pareto multiobjective optimization. Congress on Evolutionary Computation (CEC 99), 1, Piscataway:98-105, 1999.

24. R. Kudikala, K. Deb, and B. Bhattacharya. Multiobjective optimization of piezoelectric actuator placement for shape control of plates using genetic algorithms. Journal of Mechanical Design, 131:091007-1-11, 2009.

25. N.D. Lagaros and V. Papadopoulos. Optimum design of shell structures with random geometric, material and thickness imperfections. International Journal of Solids and Structures, 43:6948-6964, 2006.

26. R.H. MacNeal. A hybrid method of component mode synthesis. Computers and Structures, 1:581-601, 1971.

27. B. Paluch, M. Grédiac, and A. Faye. Combining a finite element programme and a genetic algorithm to optimize composite structures with variable thickness. Composite and Structures, 83:284-294, 2008.

28. T.H.H. Pian, D.P. Chen, and D. Kang. A new formulation of hybrid/mixed finite element. Computers \& Structures, 16:81-87, 1983.

29. E. Reissner. On a variational theorem in elasticity. Journal of Mathematics and Physics, 29:90-95, 1950.

30. E. Reissner. On a certain mixed variational theorem and a proposed application. International Journal For Numerical Methods In Engineering, 20(7):1366-1368, 1984.

31. E. Reissner. On a mixed variational theorem and on shear feformable plate theory. International Journal For Numerical Methods In Engineering, 23(2):193-198, 1986.

32. A.F. Saleeb and T.Y. Chang. On the hybrid-mixed formulation of $\mathrm{c} 0$ curved beams. Computer Method in Applied Mechanics and Engineering, 60:95-121, 1987.

33. N. Srinivas and K. Deb. Multiobjective optimization using nondominated sorting in genetic algorithms. Journal of Evolutionary Computation, 2:221-248, 1994.

34. H. Stolarski and T. Belytschko. On the equivalence of mode decomposition and mixed finite element based on the hellinger-reissner principle. part 1: Theory. Computers Methods in Applied Mechanics and Engineering, 58:249-263, 1986.

35. H. Stolarski and T. Belytschko. On the equivalence of mode decomposition and mixed finite element based on the hellinger-reissner principle. part 2: Application. Computers Methods in Applied Mechanics and Engineering, 58:265-284, 1986.

36. D.M. Tran. Component mode synthesis methods using interface modes. application to structures with cyclic symmetry. Computers and Structures, 79:209-222, 2001.

37. K. Washizu. Variational methods in elasticity and plasticity, 2nd edition. Pergamon Press, 1975.

38. P. Wriggers and C. Carstensen. Mixed Finite Element Technologies. Applied Mathematics/Computational Methods of Engineering, 2009.

39. E. Zitzler, K. Deb, and L. Thiele. Comparison of multiobjectivr evolutionary algorithms: empirical results. Technical report, Technical Report 70, Computer Engineering and Networks Laboratory (TIK), Swiss Federal Institute of Technology (ETH) Zurich, 1999.

40. E. Zitzler, E. Laumanns, and L. Thiele. Spea2: Improving the strength pareto evolutionary algorithm. Technical report, Technical Report 103, Computer Engineering and Networks Laboratory (TIK), Swiss Federal Institute of Technology (ETH) Zurich, 2001.

41. E. Zitzler and L. Thiele. Multiobjective evolutionary algorithms: a comparative case study and the strength pareto approach. evolutionary computation. IEEE transactions on Evolutionary Computation, 3(4):257-271, 1999. 